\title{
Claudin-1 and claudin-5 expression patterns differentiate lung squamous cell carcinomas from adenocarcinomas
}

\author{
Serge Paschoud ${ }^{1, *}$, Massimo Bongiovanni ${ }^{2, *}$, Jean-Claude Pache $^{2}$ and \\ Sandra Citi ${ }^{1,3}$ \\ ${ }^{1}$ Department of Molecular Biology, Faculty of Sciences, University of Geneva, Geneva, Switzerland and \\ ${ }^{2}$ Department of Pathology, Geneva University Hospital, Geneva, Switzerland and ${ }^{3}$ Department of Biology, \\ University of Padova, Padova, Italy
}

\begin{abstract}
We investigated the expression of tight junction proteins in human lung squamous cell carcinomas and adenocarcinomas by immunohistochemistry and quantitative real-time reverse transcription-polymerase chain reaction (RT-PCR). We found a statistically significant correlation between diagnosis and positivity of tumors with either claudin (CLDN)-1 or CLDN-5. Squamous cell carcinomas and basal cells of bronchial epithelium were positive for CLDN-1 and negative for CLDN-5, whereas adenocarcinomas, normal cylindrical cells and pneumocytes were positive for CLDN-5 and negative for CLDN-1, suggesting different pathways in tumor development and progression. CLDN-4 and ZO-1 staining were detected in both types of tumors, whereas cingulin (CGN) was not detected in squamous cell carcinomas. Quantitative RT-PCR was used to evaluate changes in transcript levels for a large panel of tight junction proteins. In squamous cell carcinomas, we observed statistically significant decreases in the mRNA levels of JAM-1, occludin, CLDN-3, CLDN-4, CLDN-7, CGN, ZO-2 and ZO-3, and an increase in CLDN-1 mRNA. In adenocarcinomas, when transcript levels were compared with bronchial cells, we observed statistically significant decreases in the mRNA levels of CLDN-1, CLDN-3, CLDN-4, CLDN-7, ZO-2 and ZO-3. These results indicate that characterization of tight junction protein expression in human lung tumors can be an additional diagnostic tool and provide new insights on their histogenesis.
\end{abstract}

Modern Pathology (2007) 20, 947-954; doi:10.1038/modpathol.3800835; published online 22 June 2007

Keywords: lung; adenocarcinoma; squamous cell carcinoma; claudin; cingulin; immunohistochemistry

Lung cancer is the leading cause of cancer mortality in the Western countries. Four major histological subtypes have been described: squamous cell carcinoma, adenocarcinoma, small cell and large cell carcinomas. ${ }^{1}$ Squamous cell carcinomas and adenocarcinomas account for over a half of all lung cancers, and are believed to originate, respectively, from squamous cell dysplasia/carcinoma in situ, ${ }^{1,2}$ and atypical adenomatous hyperplasia ${ }^{3}$ of lung epithelial cells. However, the precise histogenetical origin of these carcinomas is unknown: it could include pluripotent stem cells or differentiated cells and whether a common pluripo-

Correspondence: Dr S Citi, MD, PhD, Department of Molecular Biology, University of Geneva, 30, Quai Ernest Ansermet, Geneva 1211-4, Switzerland.

E-mail: sandra.citi@molbio.unige.ch

* These authors contributed equally to this work.

Received 17 March 2007; revised and accepted 23 May 2007; published online 22 June 2007 tent progenitor cell exists is questioned by different authors. Immunohistochemistry with antibodies such as p63, TTF-1 and keratins 5/6/7/14/17, CD15 and thrombomodulin is currently used to distinguish pulmonary squamous cell carcinomas from adenocarcinomas. ${ }^{4-6}$

Epithelial cells are characterized by tight junctions, which seal their apical regions in a gasket-like manner, thus preventing the free diffusion of solutes (including growth factors and pathogens) across extracellular luminal and basal compartments. Recent studies have shown that some tight junction proteins are involved in the regulation of epithelial proliferation and differentiation (reviewed by Matter et $a l^{7}$ ). Tight junctions comprise transmembrane proteins and cytoplasmic proteins. Among tight junction membrane proteins, the claudin (CLDN) family includes over 20 members, which show tissue-specific expression, and are crucial for the tight junction barrier function. 
Several studies have shown an altered expression of CLDNs in human tumors, including tumors of the gastrointestinal tract, ${ }^{8,9}$ ovary, ${ }^{10}$ pancreas, ${ }^{11}$ breast, ${ }^{12,13}$ epidermis, ${ }^{14}$ liver, ${ }^{15,16}$ uterus $^{17}$ and prostate. ${ }^{18}$ Little information is available on the expression of cytoplasmic tight junction proteins in human cancers, ${ }^{19-22}$ and only one study has been published about the immunohistochemical localization of a tight junction protein (occludin (OCLN)) in lung tumors. ${ }^{23}$

Considering the role of tight junctions in epithelial cell function, the involvement of tight junction proteins in the regulation of epithelial proliferation, and their potential usefulness as novel tools in cancer diagnosis, prognosis and treatment, ${ }^{24,25}$ it is essential to understand in detail how tumor development correlates to changes in tight junction protein expression. To address this issue, we used immunocytochemistry and the quantitative reverse transcription-polymerase chain reaction (qRT-PCR) to examine the expression of several cytoplasmic and transmembrane proteins of tight junctions in human lung squamous cell carcinomas and adenocarcinomas. Our results provide new diagnostic tools for the differential diagnosis between these tumors and suggest new insights about tumor differentiation.

\section{Materials and methods}

\section{Tissue Collection}

The study material consisted of 90 carcinomas of the lung (41 adenocarcinomas and 49 squamous cell carcinomas) obtained from the archives of the Department of Pathology at the Geneva University Hospital, in accordance with approved protocols. Among the patients, 31 were females and 59 were males, with age ranging from 30 to 85 years (median 66).

For histopathological and immunohistochemical analysis, samples were fixed in $10 \%$ buffered formalin and embedded in paraffin. Tissues showed no macroscopic evidence of necrosis or inflammation. For RNA isolation, freshly resected adenocarcinoma (11 cases), squamous carcinoma (16 cases) and normal tissue (either parenchyma or cells scraped from the lumen of bronchi or both) were stored in RNA-later (Qiagen) at $-80^{\circ} \mathrm{C}$.

\section{Immunohistochemistry}

Sections $(5 \mu \mathrm{m})$ adjacent to those used for histopathologic examination were processed for immunohistochemistry according to the manufacturer's recommendations with the following antibodies: CLDN-1 (polyclonal JAD.8, Invitrogen); CLDN-4 (clone 3E2C1, Invitrogen); CLDN-5 (clone 4C3C2, Invitrogen); ZO-1 (clone 1, Transduction Laboratories); cingulin (CGN) (clone 3G1H5, Zymed/Invitrogen). The immune reactions were carried out with the steam heat-induced epitope retrieval system, and revealed with the immunoperoxidase technique, using the Envision Kit (DakoCytomation AG, Denmark). The percentage of immunostained carcinomatous cells within the tumor was scored. For statistical purposes, cases were considered as negative when $<25 \%$ of the cells were stained.

\section{Real-Time qRT-PCR}

To prepare RNA, tissues were homogenized on ice in RLT buffer (Qiagen) using a Polytron-Aggregate homogenizer, and RNA was isolated using the RNeasy mini kit (Qiagen). cDNA was synthesized from $1.5 \mu \mathrm{g}$ total RNA, using the iScript cDNA synthesis kit (Biorad).

To quantitate transcript levels, SYBR-Green-based qRT-PCR (Applied Biosystems) and a 96-channel iCycler optical system (BioRad) were used. Triplicate samples contained $2.5 \mu \mathrm{M}$ of gene-specific primer sets, $20 \mathrm{ng}$ of cDNA, $10 \mathrm{nM}$ fluorescein dye (BioRad) and $50 \%$ volume of PCR master mix. Primer sets (Table 1 Supplementary Material) for JAM-1, OCLN, CLDN-1 to -7, cingulin, GEF-H1, PAR3, $Z O-1, Z O-2$ and $Z O-3$ were designed using Primer Express software. Primer specificity was checked by analyzing PCR products on $4 \%$ agarose gels, and by analyzing melting curves obtained by heating from 60 to $95^{\circ} \mathrm{C}$, at a rate of $0.5^{\circ} \mathrm{C}$ per $10 \mathrm{~s}$. Cycling conditions were: one cycle at $95^{\circ} \mathrm{C}$ for $10 \mathrm{~min}, 40$ cycles of amplification (each $95^{\circ} \mathrm{C}$ for $15 \mathrm{~s}$ and $60^{\circ} \mathrm{C}$ for $45 \mathrm{~s}$ ). To analyze the fluorescence signal, a threshold cycle $\left(C_{\mathrm{t}}\right)$ was determined, using the exponential growth phase and the baseline signal from fluorescence vs cycle number plots. $C_{\mathrm{t}} \mathrm{s}$ were normalized to the level of expression of housekeeping genes. Following data analysis with GeNorm v3.2 (http://medgen.ugent.be/ jvdesomp/ genorm) software, the genes, succinate dehydrogenase complex, subunit A, flavoprotein (Fp) (SDHA, NM_004168), ubiquitin (UBC, M26880) and tyrosine 3-monooxygenase/tryptophan 5-monooxygenase activation protein, zeta polypeptide (YWHAZ, NM_003406), were found to be the most stable internal control genes, and were chosen for the normalization of experimental values, using the geometric averaging method. ${ }^{26}$

\section{Statistical Analysis}

Associations between clinicopathologic parameters and CLDN-1 and CLDN-5 expression were analyzed by the $\chi^{2}$ test. $P<0.05$ was considered statistically significant.

The statistical significance of the difference in transcript levels between cancerous and control tissues was assessed with the two-tailed one-sample $t$-test, using Prism software (GraphPad Software Inc.). 


\section{Results}

Immunolocalization of CLDN-1, CLDN-4, CLDN-5, ZO-1 and CGN in Lung Adenocarcinomas and Squamous Cell Carcinomas

Sections of normal bronchial tissue, lung parenchyma, squamous cell carcinoma and adenocarcinoma were labeled with antibodies against three members of the CLDN family of transmembrane tight junction proteins (CLDN-1, CLDN-4, CLDN-5), and two cytoplasmic tight junction proteins (ZO-1 and CGN) (Figure 1 and Table 1). Intense membrane labeling for CLDN-1 was detected in the basal cells of normal bronchial epithelium (double arrows in Figure 1a), whereas cylindrical cells displayed weak or no labeling along their lateral borders (arrowhead in Figure 1a), suggesting that differentiation of basal cells into differentiated cylindrical cells is associated with a downregulation of CLDN-1 expression. Cells undergoing squamous cell dysplasia showed strong CLDN-1 immunolabeling (arrow in Figure 1a), whereas CLDN-5 and CGN labeling were undetectable in these cells (not shown). Conversely, CLDN-1 labeling was not detected in pneumocytes of normal parenchyma (arrowhead in Figure 1b). No CLDN-1 labeling was detected in adenocarcinomas
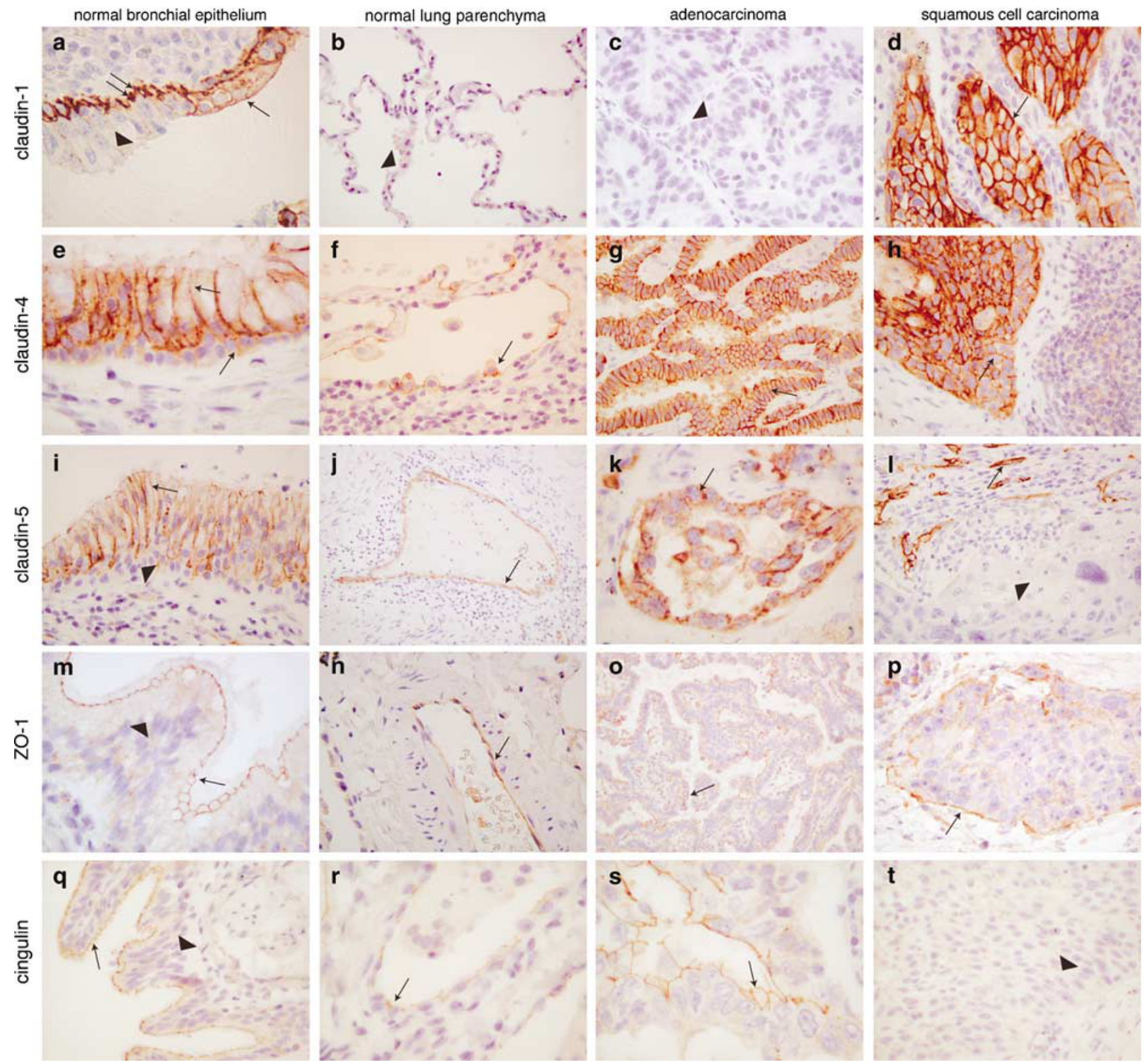

Figure 1 Immunolocalization of CLDN-1 (a-d), CLDN-4 (e-h), CLDN-5 (i-l), ZO-1 (m-p) and CGN (q-t) in normal human bronchial epithelium (a, e, $\mathbf{i}, \mathbf{m}, \mathbf{q})$, normal parenchyma $(\mathbf{b}, \mathbf{f}, \mathbf{j}, \mathbf{n}, \mathbf{r})$, adenocarcinoma $(\mathbf{c}, \mathbf{g}, \mathbf{k}, \mathbf{o}, \mathbf{s})$ and squamous cell carcinoma $(\mathbf{d}, \mathbf{h}, \mathbf{l}, \mathbf{p}, \mathbf{t})$. Arrows indicate positive staining, and arrowheads indicate negative staining. In the case of normal parenchyma, either pneumocytes $(\mathbf{b}, \mathbf{f}, \mathbf{r})$ or blood vessels $(\mathbf{j}, \mathbf{n})$ are shown. Sections were observed at original magnifications between $\times 10$ and $\times 40$. Note the lack of staining of adenocarcinoma by CLDN-1 (c), and of squamous cell carcinoma by CLDN-5 (l) and CGN (t). 
Table 1 Expression of claudin-1, -4, -5, ZO-1 and cingulin in normal and neoplastic human lung tissues

\begin{tabular}{|c|c|c|c|c|c|c|}
\hline & & Claudin-1 & Claudin-4 & Claudin-5 & $Z O-1$ & Cingulin \\
\hline \multirow[t]{2}{*}{ Bronchial mucosa } & Cylindrical cells & - & + & + & + & + \\
\hline & Basal cells & + & + & - & - & - \\
\hline \multirow{4}{*}{ Peripheral parenchyma } & Pneumocytes & - & + & + & + & + \\
\hline & Endothelial cells & - & - & + & + & - \\
\hline & Fibroblasts & - & - & - & - & - \\
\hline & Lymphocytes & - & - & - & - & - \\
\hline \multirow[t]{2}{*}{ Carcinoma } & Adenocarcinoma & - & + & + & + & + \\
\hline & Squamous cell carcinoma & + & + & - & + & - \\
\hline
\end{tabular}

(arrowhead in Figure 1c). Conversely, squamous cell carcinomas showed intense CLDN-1 immunolabeling, which was distributed all around the cell peripheries (arrow in Figure 1d). In summary, strong CLDN-1 immunolabeling was detected in normal basal bronchial epithelial cells and in squamous cell carcinomas, whereas no staining was detected in pneumocytes and adenocarcinoma tissue, as well as fibroblasts, endothelial cells and lymphocytes of peripheral parenchyma (Table 1).

CLDN-4 labeling was detected in the basal cells of the bronchial epithelium, along the lateral membrane of cylindrical cells (arrows in Figure 1e), and in pneumocytes (arrow in Figure 1f). Strong staining was detected both in adenocarcinomas (arrow in Figure 1g) and squamous cell carcinomas (arrow in Figure 1f). Endothelial cells, lymphocytes and fibroblasts were negative (Table 1).

The expression and localization of CLDN-5 were clearly distinct from those of CLDN-1 and CLDN-4. In normal bronchial epithelium, cylindrical cells showed strong labeling along lateral borders (arrow in Figure 1i), whereas basal cells were negative (arrowhead in Figure 1i). Endothelial cells (arrow in Figure 1j) and pneumocytes of the peripheral parenchyma were positive (Table 1). Strong membrane labeling of CLDN-5 was detected in adenocarcinomas (arrow in Figure 1k). In contrast, squamous cell carcinomas showed no CLDN-5 labeling (arrowhead in Figure 1l), whereas neighboring blood vessels were positive (arrow in Figure 1l). In summary, CLDN-5 was detected in cylindrical cells and adenocarcinomas, but not in basal cells and squamous cell carcinomas (Table 1).

In normal bronchial epithelium, the immunolocalization of the cytoplasmic tight junction proteins ZO-1 (Figure 1m) and CGN (Figure 1q) was similar, with the accumulation of labeling in a honeycomblike pattern at the apicolateral border of epithelial cells, facing the lumen, corresponding to tight junctions (arrows in Figure $1 \mathrm{~m}$ and q). Basal cells were negative for CGN and ZO-1 (arrowheads in Figure $1 \mathrm{~m}$ and q). Endothelial cells of blood vessels within the parenchyma were positive for ZO-1 (arrow in Figure 1n), and negative for CGN (Table 1), whereas pneumocytes were positive for CGN (arrow in Figure 1r) and ZO-1 (Table 1). In adenocarcinomas, ZO-1 and CGN immunolabeling was detected in the glandular component, and was distributed in the region of the cells facing residual true or virtual lumens (arrows in Figure 1o and s). Squamous cell carcinomas showed diffuse ZO-1 staining, which was more intense at the border between tumor tissue and stroma (arrow in Figure 1p). In contrast, CGN immunolabeling was reduced in squamous cell carcinoma (arrowhead in Figure 1t), but occasionally detected in poorly differentiated cases in superficial neoplastic cells facing a virtual lumen. In summary, both ZO-1 and CGN were detected in cylindrical cells, pneumocytes and adenocarcinomas, and ZO-1 was also detected in endothelial cells and squamous cell carcinomas (Table 1).

The expression of CLDN-1 and CLDN-5 was correlated to the clinicopathological characteristics of the lung carcinomas. A statistically significant correlation was found between CLDN-1 expression and squamous cell carcinoma (Table 2), and between CLDN-5 expression and adenocarcinomas (Table 3). However, there was no significant correlation between the staining for these markers and parameters such as pathologic $\mathrm{T}$ stage, $\mathrm{N}$ stage and histologic differentiation (Table 2 and Table 3 ).

\section{Changes in Transcript Levels for Tight Junction Proteins in Lung Squamous Cell Carcinomas and Adenocarcinomas}

To confirm and extend the observations on the altered patterns of tight junction protein expression in lung squamous cell carcinomas and adenocarcinomas, transcript levels for 14 different tight junction-associated proteins, including the ones analyzed by immunocytochemistry, were measured by qRT-PCR (Table 4). Transcript levels were expressed as percentages, taking as $100 \%$, the transcript levels of control normal tissue obtained from the same patient. Either parenchyma or scraped cells from normal bronchi, or both were used as a control for each sample of tumor tissue (Table 4).

Squamous cell carcinomas showed statistically significant decreases in mRNA levels ( $v s$ bronchial cells) for JAM-1 (which was decreased by $\sim 45 \%$ ) OCLN ( 67\%), CLDN-3 ( 95\%), CLDN-4 ( 65\%), 
Table 2 Relationship between claudin-1 protein expression and clinicopathological characteristics of lung carcinomas

\begin{tabular}{lcc}
\hline & $\begin{array}{c}\text { Claudin-1 } \\
\text { expression }\end{array}$ & P-value \\
\hline Diagnosis & & \\
$\quad$ Adenocarcinoma & $7 / 41(17 \%)$ & $0.0000^{*}$ \\
Squamous cell carcinoma & $38 / 49(77.5 \%)$ & \\
& & \\
p stage (squamous cell carcinoma) & & \\
T1 & $7 / 9(77.7 \%)$ & 0.9332 \\
T2 & $26 / 34(76.5 \%)$ & \\
T3 & $5 / 6(83.3 \%)$ & \\
& & \\
$p N$ stage (squamous cell carcinoma) & $22 / 29(75.9 \%)$ & 0.5312 \\
N0 & $9 / 10(90 \%)$ & \\
N1 & $7 / 10(70 \%)$ & \\
N2 & & \\
Histology (squamous cell carcinoma) & & \\
Well differentiated & $5 / 6(83.3 \%)$ & 0.3627 \\
Moderately differentiated & $18 / 21(85.7 \%)$ & \\
Poorly differentiated & $15 / 22(68.2 \%)$ & \\
\hline
\end{tabular}

*Statistically significant $P<0.05$.

Table 3 Relationship between claudin-5 protein expression and clinicopathological characteristics of lung carcinomas

\begin{tabular}{lcc}
\hline & $\begin{array}{c}\text { Claudin-5 } \\
\text { expression }\end{array}$ & P-value \\
\hline Diagnosis & & \\
$\quad$ Adenocarcinoma & $27 / 41(63.4 \%)$ & 0.0084 * \\
Squamous cell carcinoma & $17 / 49(34.6 \%)$ & \\
pT stage (adenocarcinoma) & & \\
T1 & $8 / 13(61.5 \%)$ & 0.8782 \\
T2 & $15 / 24(62.5 \%)$ & \\
T3 & $3 / 4(75 \%)$ & \\
& & \\
pN stage (adenocarcinoma) & $18 / 25(72 \%)$ & 0.3554 \\
N0 & $6 / 9(66.6 \%)$ & \\
N1 & $3 / 7(42.8 \%)$ & \\
N2 & & \\
Histology (adenocarcinoma) & $15 / 22(68.2 \%)$ & 0.5117 \\
Well differentiated & $5 / 7(71.4 \%)$ & \\
Moderate differentiated & $6 / 12(50 \%)$ & \\
Poorly differentiated &
\end{tabular}

*Statistically significant $P<0.05$.

CLDN-7 ( 76\%), CGN ( 69\%), ZO-2 ( 71\%) and $Z O-3(\sim 97 \%)$. When parenchyma was used as a control, squamous cell carcinomas showed a significant decrease in mRNA levels for OCLN ( 83\%), CLDN-5 ( 84\%), CGN ( 76\%), GEF-H1 ( $\sim 69 \%), Z O-1(\sim 76 \%), Z O-2(\sim 70 \%)$ and $Z O-3$ $(\sim 60 \%)$. The only proteins for which a statistically significant increase in mRNA levels was found in squamous cell carcinoma (vs parenchyma) were $C L D N-1$ ( $\sim 7$-fold increase) and CLDN-5 ( $\sim 8$-fold increase) ( $v s$ bronchial cells). Although the increase in $C L D N-1$ transcript levels was correlated to an increased protein detection by immunocytochemistry, no $C L D N-5$ was detected in squamous cell carcinoma by antibody staining (Figure 1). Increases were also observed in the transcript levels of $C L D N$ 1, CLDN-2 and GEF-H1, but they were not statistically significant in our sample pool (Table 4).

For adenocarcinomas, the relative levels of mRNA of tight junction proteins were somewhat different (except for JAM-1, OCLN, CGN, PAR-3 and ZO-2), depending on whether parenchyma or scraped bronchial cells were used as a control. As the results with bronchial cells were in better agreement with the results obtained by immunocytochemistry, bronchial cells may represent the most appropriate normal control tissue. Notably, when using bronchial cells as a control, we observed a statistically significant decrease in the transcript levels for $C L D N-1 \quad(\sim 58 \%), C L D N-3 \quad(\sim 71 \%), C L D N-4$ ( 47\%), CLDN-7 ( 57\%), ZO-2 ( 72\%) and $Z O-3$ $(\sim 82 \%)$. A statistically significant increase was observed for GEF-H1 mRNA ( 2-fold), whereas expression of $C G N$ and $Z O-1$ was similar between adenocarcinoma and bronchial cells (Table 4). In agreement with the immunocytochemistry results, $C L D N-5$ mRNA levels were increased $(\sim 33$-fold $)$ compared with bronchial cells, but, surprisingly, were decreased $(\sim 87 \%)$ compared with parenchyma. This latter discrepancy could be due to the abundance of $C L D N-5$ transcript in highly vascularized parenchyma. The increase in $C L D N-4$ mRNA ( 2.6-fold) compared with parenchyma was correlated with the immunocytochemistry observations, whereas the increase in $C L D N-1$ mRNA was not, suggesting that $C L D N-1$ mRNA translation may be repressed in adenocarcinomas.

\section{Discussion}

This study provides the first analysis of the expression of a large panel of tight junction proteins in human lung adenocarcinomas and squamous cell carcinomas, using both immunocytochemistry and qRT-PCR. We demonstrate that CLDN-1, CLDN-5 and CGN are differently expressed in these two tumors, and their localization in normal bronchial tissue suggests hypotheses on the histogenesis of these tumors. Furthermore, we show that expression of several other tight junction proteins is altered in human lung tumors.

CLDN-1 is expressed in a large number of tissues, and plays a crucial role in the barrier function of skin. ${ }^{27}$ Here we showed that CLDN-1 expression is high in lung squamous cell carcinomas (both by protein detection and transcript quantification), but reduced or undetected in adenocarcinomas. Interestingly, an increase in CLDN-1 expression was observed in keratinized regions of squamous cell carcinoma of the epidermis, ${ }^{14}$ and in colon cancers, ${ }^{28}$ whereas a decrease or loss of expression was reported in breast cancer cell lines ${ }^{29,30}$ and tumors. ${ }^{31}$ These findings suggest that a squamous cell tumor phenotype is more likely to be correlated with high 
Table 4 Quantitative analysis of transcript levels for tight junction proteins by qRT-PCR

\begin{tabular}{|c|c|c|c|c|}
\hline Protein & $\begin{array}{c}\text { Squamous c.c. vs } \\
\text { parenchyma }(\mathrm{n}=10)\end{array}$ & $\begin{array}{c}\text { Squamous } \text { C.c. vs } \\
\text { bronchial cell }(\mathrm{n}=7)\end{array}$ & $\begin{array}{l}\text { Adenocarcinoma vs } \\
\text { parenchyma }(\mathrm{n}=7)\end{array}$ & $\begin{array}{l}\text { Adenocarcinoma vs } \\
\text { bronchial cell }(\mathrm{n}=6)\end{array}$ \\
\hline JAM-1 & $104 \pm 10$ & $56 \pm 11^{*}$ & $142 \pm 33$ & $81 \pm 17$ \\
\hline Occludin & $17 \pm 4^{*}$ & $33 \pm 13$ * & $114 \pm 36$ & $155 \pm 37$ \\
\hline Claudin-1 & $708 \pm 175^{*}$ & $289 \pm 195$ & $612 \pm 275$ & $42 \pm 18^{*}$ \\
\hline Claudin-2 & $113 \pm 47$ & $274 \pm 132$ & $86 \pm 48$ & $643 \pm 401$ \\
\hline Claudin-3 & $118 \pm 80$ & $4 \pm 2 *$ & $765 \pm 358$ & $29 \pm 8^{*}$ \\
\hline Claudin-4 & $105 \pm 29$ & $35 \pm 14^{*}$ & $263 \pm 56^{*}$ & $63 \pm 14^{*}$ \\
\hline Claudin-5 & $16 \pm 8^{*}$ & $821 \pm 256^{*}$ & $13 \pm 3^{*}$ & $3384 \pm 1305$ \\
\hline Claudin-7 & $94 \pm 18$ & $24 \pm 9^{*}$ & $250 \pm 55^{*}$ & $43 \pm 9^{*}$ \\
\hline Cingulin & $24 \pm 9^{*}$ & $31 \pm 19 *$ & $139 \pm 60$ & $91 \pm 25$ \\
\hline GEF-H1 & $31 \pm 6^{*}$ & $188 \pm 79$ & $63 \pm 17$ & $220 \pm 38^{*}$ \\
\hline PAR-3 & $116 \pm 12$ & $97 \pm 36$ & $77 \pm 20$ & $92 \pm 29$ \\
\hline ZO-1 & $24 \pm 3^{*}$ & $121 \pm 19$ & $40 \pm 11^{*}$ & $113 \pm 25$ \\
\hline ZO-2 & $30 \pm 6^{*}$ & $29 \pm 6^{*}$ & $57 \pm 12^{*}$ & $28 \pm 5^{*}$ \\
\hline $\mathrm{ZO}-3$ & $40 \pm 12 *$ & $3 \pm 2$ * & $302 \pm 66^{*}$ & $18 \pm 4^{*}$ \\
\hline
\end{tabular}

Values are expressed as percentages ( \pm s.e.m., standard error of the mean), taking as $100 \%$ the level of expression of control tissue. Squamous c.c. = squamous cell carcinoma.

* Statistically significant $P<0.05$.

CLDN-1 expression, whereas tumors with glandular components are more likely to lose CLDN-1 expression. In addition, our observation that in normal bronchial epithelium CLDN-1 is abundant in basal cells, but scarce or absent in cylindrical cells and pneumocytes raises the hypothesis that squamous cell carcinomas arise either from uncontrolled proliferation and lack of normal differentiation of the basal cells of the bronchial epithelium or from the reversion of differentiated cells to this phenotype. As $C L D N-1$ has been implicated in epithelial-mesenchymal transition, possibly through the modulation of the $\beta$-catenin/Tcf signaling pathway ${ }^{28}$ it is possible that $C L D N-1$ dysregulation may be part of the molecular pathway that leads to the development of squamous cell carcinomas.

The pattern of CLDN-5 expression in normal bronchial epithelium and in tumors was essentially opposite to that of CLDN-1, with high expression in normal cylindrical cells, pneumocytes and adenocarcinomas, and low or undetectable expression in basal cells and squamous cell carcinomas. These results indicate not only that CLDN-5 could be used as an additional diagnostic tool but also that the differentiation pathway of adenocarcinomas is distinct of that of squamous cell carcinomas. Little information is available on CLDN-5 expression in human cancers, apart from a reported decrease in hepatocellular and renal carcinomas. ${ }^{32}$ Previous studies indicated that CLDN-5 is specifically expressed in endothelial cells. ${ }^{33,34}$ We confirmed CLDN-5 expression in blood vessels, and we also demonstrated its expression in bronchial cells and pneumocytes. The high level of CLDN-5 expression in endothelia may explain why mRNA levels of $C L D N-5$ were decreased in adenocarcinomas (which contain mostly epithelial cells), when compared with lung parenchyma (where abundant blood vessels are present) (Table 4). On the other hand, $C L D N-5$ mRNA levels were high in adenocarcinomas, when compared with bronchial cells (Table 4). In squamous cell carcinomas, $C L D N-5$ mRNA appeared to be abundant (only when compared with scraped bronchial cells), despite the lack of CLDN-5 immunoreactivity in these tumors. This discrepancy could be explained by a downregulation of CLDN-5 expression at the translational level. As different results were obtained in some cases depending on whether parenchyma or scraped bronchial cells were used as a control tissue, results using qRTPCR should be interpreted with caution. A careful choice of the control normal tissue, as well as the use of multiple internal control housekeeping genes is necessary. Results should be confirmed by immunocytochemistry, which provides essential information about the cellular and subcellular distribution of proteins. In this regard, it is noteworthy that in lung normal and tumor epithelial cells CLDN-1, -4 and -5 were not restricted to apicolateral junctions, as CGN and ZO-1, but were detected throughout the membrane surface, raising the hypothesis that CLDNs may play additional roles, besides their canonical role in the tight junction barrier.

qRT-PCR indicated a decreased expression of $C L D N-3$ and $C L D N-7$ both in adenocarcinomas and squamous cell carcinomas, when compared with bronchial cells. CLDN-4 mRNA was decreased in squamous cell carcinomas, but the decrease in mRNA (Table 4) did not correlate with a decreased protein expression (Figure 1). This could be due to an increased mRNA or protein stability. CLDN-3 and CLDN-4 are upregulated in ovarian and uterine cancers, ${ }^{10,17,35,36}$ and CLDN-4 expression has been exploited for therapeutic purposes. ${ }^{24}$ CLDN-7 expression is decreased in breast carcinomas, ${ }^{13,37}$ but 
upregulated in gastric carcinomas. ${ }^{9}$ With regards to other tight junction membrane proteins, we observed a statistically significant decrease in JAM-1 transcript levels in squamous cell carcinoma, when compared with normal bronchial cells, and in OCLN mRNA in squamous cell carcinoma. This latter result is in agreement with one previous immunohistochemistry study of OCLN expression in lung carcinomas. ${ }^{23}$

When analyzing the expression of cytoplasmic tight junction proteins, we observed significant decreases in the expression of $Z O-2$ and $Z O-3$ in both squamous cell carcinomas and adenocarcinomas, when compared with bronchial cells, and $\mathrm{ZO}-2$ also when compared with parenchyma. However, the expression of the related protein ZO-1 was maintained. CGN was expressed in lung adenocarcinomas, but was significantly reduced in squamous cell carcinomas, and present only in superficial neoplastic cells facing a virtual lumen. These observations, together with our previous report on high CGN expression in colon adenocarcinomas, ${ }^{20}$ indicate that CGN could be used as an additional diagnostic tool to distinguish adenocarcinomas from other types of epithelial cancers, and to identify glandular differentiation in poorly differentiated carcinomas. The mRNA levels for the RhoA activator GEF-H1 were decreased in squamous cell carcinomas, when compared with parenchyma, and increased in adenocarcinomas, when compared with bronchial cells. Interestingly, GEF-H1 upregulation is induced by mutant p53, and leads to increased proliferation. ${ }^{38}$

Taken together, our results show that the composition of the tight junction multiprotein complex is considerably altered following cancer development in lungs. In a few instances, dramatic protein upregulation was observed (most notably, CLDN-1 in squamous cell carcinomas and CLDN-5 in adenocarcinomas), and, more often, a decrease in expression was detected. Although it is unclear how these changes affect the structure and function of tight junctions in carcinomas, it is reasonable to hypothesize that a downregulation of the adhesive function of tight junctions may lead to increased invasion and metastasis. In addition, downregulation of some tight junction proteins is known to increase epithelial proliferation in invertebrate ${ }^{39}$ and vertebrate model systems. ${ }^{40-42}$ The disruption of the tight junction multiprotein complex may also affect the establishment and maintenance of the polarized phenotype. Although it remains unclear if and how the modified expression of any given specific tight junction protein is associated with cancer pathogenesis, our study suggests that it may be useful to understand cancer histogenesis. Further work should be carried out to understand how molecular abnormalities in the expression of tight junction proteins could contribute to tumorigenesis, and to expand their use as tools for cancer diagnosis, prognosis and treatment.

\section{Acknowledgements}

This work was supported by Grants from the Swiss Cancer League (OCS-01390-08-2003 and OCS01916-08-2006), the Swiss National Science Foundation, by the State of Geneva and by the Italian Ministry for Scientific Research (ex-60\%). We thank Monique Coassin and Lionel Jond for technical assistance, and Gioacchino Leandro (IRCSS, Castellana Grotte, Italy) for help with statistics.

\section{References}

1 Travis WD, Colby TV, Corrin B, et al. Histologic Typing of Lung and Pleural Tumors. Springer: Berlin, 1999.

2 Saccomanno G, Archer VE, Auerbach O, et al. Development of carcinoma of the lung as reflected in exfoliated cells. Cancer 1974;33:256-270.

3 Mori M, Rao SK, Popper HH, et al. Atypical adenomatous hyperplasia of the lung: a probable forerunner in the development of adenocarcinoma of the lung. Mod Pathol 2001;14:72-84.

4 Kalhor N, Zander DS, Liu J. TTF-1 and p63 for distinguishing pulmonary small-cell carcinoma from poorly differentiated squamous cell carcinoma in previously pap-stained cytologic material. Mod Pathol 2006;19:1117-1123.

5 Camilo R, Capelozzi VL, Siqueira SA, et al. Expression of p63, keratin 5/6, keratin 7 , and surfactant-A in non-small cell lung carcinomas. Hum Pathol 2006;37: 542-546.

6 Pritchard SA, Howat AJ, Edwards JM. Immunohistochemical panel for distinction between squamous cell carcinoma, adenocarcinoma and mesothelioma. Histopathology 2003;43:197-199.

7 Matter K, Aijaz S, Tsapara A, et al. Mammalian tight junctions in the regulation of epithelial differentiation and proliferation. Curr Opin Cell Biol 2005;17: 453-458.

8 Miwa N, Furuse M, Tsukita S, et al. Involvement of claudin-1 in the beta-catenin/Tcf signaling pathway and its frequent upregulation in human colorectal cancers. Oncol Res 2000;12:469-476.

9 Johnson AH, Frierson HF, Zaika A, et al. Expression of tight-junction protein claudin-7 is an early event in gastric tumorigenesis. Am J Pathol 2005;167:577-584.

10 Rangel LB, Agarwal R, D’Souza T, et al. Tight junction proteins claudin-3 and claudin-4 are frequently overexpressed in ovarian cancer but not in ovarian cystadenomas. Clin Cancer Res 2003;9:2567-2575.

11 Michl P, Barth C, Buchholz M, et al. Claudin-4 expression decreases invasiveness and metastatic potential of pancreatic cancer. Cancer Res 2003;63: 6265-6271.

12 Kramer F, White K, Kubbies M, et al. Genomic organization of claudin-1 and its assessment in hereditary and sporadic breast cancer. Hum Genet 2000;107:249-256.

13 Kominsky SL, Argani P, Korz D, et al. Loss of the tight junction protein claudin-7 correlates with histological grade in both ductal carcinoma in situ and invasive ductal carcinoma of the breast. Oncogene 2003;22: 2021-2033.

14 Morita K, Tsukita S, Miyachi Y. Tight junctionassociated proteins (occludin, ZO-1, claudin-1, 
claudin-4) in squamous cell carcinoma and Bowen's disease. Br J Dermatol 2004;151:328-334.

15 Cheung ST, Leung KL, Ip YC, et al. Claudin-10 expression level is associated with recurrence of primary hepatocellular carcinoma. Clin Cancer Res 2005;11:551-556.

16 Lodi C, Szabo E, Holczbauer A, et al. Claudin-4 differentiates biliary tract cancers from hepatocellular carcinomas. Mod Pathol 2006;19:460-469.

17 Santin AD, Zhan F, Cane S, et al. Gene expression fingerprint of uterine serous papillary carcinoma: identification of novel molecular markers for uterine serous cancer diagnosis and therapy. Br J Cancer 2005; 92:1561-1573.

18 Long H, Crean CD, Lee WH, et al. Expression of Clostridium perfringens enterotoxin receptors claudin3 and claudin-4 in prostate cancer epithelium. Cancer Res 2001;61:7878-7881.

19 Hoover KB, Liao SY, Bryant PJ. Loss of the tight junction MAGUK ZO-1 in breast cancer: relationship to glandular differentiation and loss of heterozygosity. Am J Pathol 1998;153:1767-1773.

20 Citi S, Amorosi A, Franconi F, et al. Cingulin, a specific protein component of tight junctions, is expressed in normal and neoplastic human epithelial tissues. Am J Pathol 1991;138:781-789.

21 Chlenski A, Ketels KV, Tsao MS, et al. Tight junction protein ZO-2 is differentially expressed in normal pancreatic ducts compared to human pancreatic adenocarcinoma. Int J Cancer 1999;82:137-144.

22 Martin TA, Watkins G, Mansel RE, et al. Loss of tight junction plaque molecules in breast cancer tissues is associated with a poor prognosis in patients with breast cancer. Eur J Cancer 2004;40:2717-2725.

23 Tobioka $\mathrm{H}$, Tokunaga $\mathrm{Y}$, Isomura $\mathrm{H}$, et al. Expression of occludin, a tight-junction-associated protein, in human lung carcinomas. Virchows Arch 2004;445:472-476.

24 Michl P, Buchholz M, Rolke M, et al. Claudin-4: a new target for pancreatic cancer treatment using Clostridium perfringens enterotoxin. Gastroenterology 2001; 121:678-684.

25 Hewitt KJ, Agarwal R, Morin PJ. The claudin gene family: expression in normal and neoplastic tissues. BMC Cancer 2006;6:186.

26 Vandesompele J, De Preter K, Pattyn F, et al. Accurate normalization of real-time quantitative RT-PCR data by geometric averaging of multiple internal control genes. Genome Biol 2002;3:RESEARCH0034.1-.12.

27 Furuse M, Hata M, Furuse K, et al. Claudin-based tight junctions are crucial for the mammalian epidermal barrier: a lesson from claudin-1-deficient mice. J Cell Biol 2002;156:1099-1111.

28 Dhawan P, Singh AB, Deane NG, et al. Claudin-1 regulates cellular transformation and metastatic behavior in colon cancer. J Clin Invest 2005;115:1765-1776.

29 Swisshelm K, Machl A, Planitzer S, et al. SEMP1, a senescence-associated cDNA isolated from human mammary epithelial cells, is a member of an epithelial membrane protein superfamily. Gene 1999;226: 285-295.

30 Hoevel T, Macek R, Swisshelm K, et al. Reexpression of the tight junction protein CLDN1 induces apoptosis in breast tumor spheroids. Int J Cancer 2004;108: 374-383.

31 Tokes AM, Kulka J, Paku S, et al. Claudin-1, -3 and -4 proteins and mRNA expression in benign and malignant breast lesions: a research study. Breast Cancer Res 2005;7:R296-R305.

32 Soini Y. Expression of claudins 1, 2, 3, 4, 5 and 7 in various types of tumours. Histopathology 2005;46: $551-560$.

33 Morita K, Sasaki H, Furuse M, et al. Endothelial claudin: claudin-5/TMVCF constitutes tight junction strands in endothelial cells. J Cell Biol 1999;147: 185-194.

34 Nitta T, Hata M, Gotoh S, et al. Size-selective loosening of the blood-brain barrier in claudin-5-deficient mice. J Cell Biol 2003;161:653-660.

35 Heinzelmann-Schwarz VA, Gardiner-Garden M, Henshall SM, et al. Overexpression of the cell adhesion molecules DDR1, Claudin 3, and Ep-CAM in metaplastic ovarian epithelium and ovarian cancer. Clin Cancer Res 2004;10:4427-4436.

36 Agarwal R, D'Souza T, Morin PJ. Claudin-3 and claudin-4 expression in ovarian epithelial cells enhances invasion and is associated with increased matrix metalloproteinase-2 activity. Cancer Res 2005; 65:7378-7385.

37 Sauer T, Pedersen MK, Ebeltoft K, et al. Reduced expression of Claudin-7 in fine needle aspirates from breast carcinomas correlate with grading and metastatic disease. Cytopathology 2005;16:193-198.

38 Mizuarai S, Yamanaka K, Kotani H. Mutant p53 induces the GEF-H1 oncogene, a guanine nucleotide exchange factor-H1 for RhoA, resulting in accelerated cell proliferation in tumor cells. Cancer Res 2006;66: 6319-6326.

39 Woods DF, Bryant PJ. The discs-large tumor suppressor gene of Drosophyla encodes a guanylate kinase homolog localized at septate junctions. Cell 1991;66: 451-464.

40 Reichert M, Muller T, Hunziker W. The PDZ domains of zonula occludens-1 induce an epithelial to mesenchymal transition of Madin-Darby canine kidney I cells. Evidence for a role of beta-catenin/Tcf/Lef signaling. J Biol Chem 2000;275:9492-9500.

41 Kausalya PJ, Phua DC, Hunziker W. Association of ARVCF with zonula occludens (ZO)-1 and ZO-2: binding to PDZ-domain proteins and cell-cell adhesion regulate plasma membrane and nuclear localization of ARVCF. Mol Biol Cell 2004;15:5503-5515.

42 Guillemot L, Citi S. Cingulin regulates claudin-2 expression and cell proliferation through the small GTPase RhoA. Mol Biol Cell 2006;17:3569-3577.

Supplementary Information accompanies the paper on Modern Pathology website (http://www.nature.com/ modpathol) 